\title{
Multiexon deletion alleles of ATF6 linked to achromatopsia
}

\author{
Eun-Jin Lee, ${ }^{1,2,3}$ Wei-Chieh Jerry Chiang, ${ }^{2,4}$ Heike Kroeger, ${ }^{2,5}$ Chloe Xiaoke Bi, ${ }^{2}$ Daniel L. Chao, ${ }^{1}$ \\ Dorota Skowronska-Krawczyk, ${ }^{1}$ Rebecca R. Mastey, ${ }^{6}$ Stephen H. Tsang, ${ }^{7}$ Leon Chea, ${ }^{8}$ Kyle Kim, ${ }^{2}$ \\ Scott R. Lambert, ${ }^{3}$ Julia M.D. Grandjean, ${ }^{9}$ Britta Baumann, ${ }^{10}$ Isabelle Audo, ${ }^{11,12}$ Susanne Kohl, ${ }^{10}$ \\ Anthony T. Moore, ${ }^{13}$ R. Luke Wiseman, ${ }^{9}$ Joseph Carroll, ${ }^{6}$ and Jonathan H. Lin $^{3,8,14}$ \\ 'Department of Ophthalmology, Shiley Eye Institute, and 'Department of Pathology, UCSD, San Diego, California, USA. \\ ${ }^{3}$ Department of Ophthalmology, Stanford University, Stanford, California, USA. ${ }^{4}$ Developmental Neurobiology Unit, \\ Okinawa Institute of Science and Technology Graduate University, Okinawa, Japan. ${ }^{5}$ Department of Cellular Biology, \\ University of Georgia, Athens, Georgia, USA. ${ }^{6}$ Department of Ophthalmology and Visual Sciences, Medical College of \\ Wisconsin, Milwaukee, Wisconsin, USA. 'Departments of Ophthalmology and Pathology and Cell Biology, Vagelos \\ College of Physicians and Surgeons, Columbia University, New York, New York, USA. ${ }^{8}$ Department of Pathology, Stanford \\ University, Stanford, California, USA. ${ }^{9}$ Department of Molecular Medicine, The Scripps Research Institute, San Diego, \\ California, USA. ${ }^{10}$ Institute for Ophthalmic Research, Centre for Ophthalmology, University of Tübingen, Tübingen, \\ Germany. ${ }^{11}$ Sorbonne Université, INSERM, CNRS, Institut de la Vision, Paris, France. ${ }^{12}$ CHNO des Quinze-Vingts, DHU Sight \\ Restore, INSERM-DGOS CIC1423, Paris, France. ${ }^{13}$ Department of Ophthalmology, UCSF, San Francisco, California, USA. \\ ${ }^{14}$ VA Palo Alto Healthcare System, Palo Alto, California, USA.
}

Achromatopsia (ACHM) is an autosomal recessive disease that results in severe visual loss. Symptoms of ACHM include impaired visual acuity, nystagmus, and photoaversion starting from infancy; furthermore, ACHM is associated with bilateral foveal hypoplasia and absent or severely reduced cone photoreceptor function on electroretinography. Here, we performed genetic sequencing in 3 patients from 2 families with ACHM, identifying and functionally characterizing 2 mutations in the activating transcription factor 6 (ATF6) gene. We identified a homozygous deletion covering exons 8-14 of the ATF6 gene from 2 siblings from the same family. In another patient from a different family, we identified a heterozygous deletion covering exons 2 and 3 of the ATF6 gene found in trans with a previously identified ATF6 c.970C >T (p.Arg324Cys) ACHM disease allele. Recombinant ATF6 proteins bearing these exon deletions showed markedly impaired transcriptional activity by qPCR and RNA-Seq analysis compared with WT-ATF6. Finally, RNAscope revealed that $A T F 6$ and the related $A T F 6 B$ transcripts were expressed in cones as well as in all retinal layers in normal human retina. Overall, our data identify loss-of-function ATF6 disease alleles that cause human foveal disease.

Conflict of interest: $J C$ has research support from Optovue, AGTC, and MeiraGTx; is a paid consultant for MeiraGTx; and has equity interest in Translational Imaging Innovations.

Copyright: (ㄷ) 2020, American Society for Clinical Investigation.

Submitted: December 27, 2019

Accepted: March 4, 2020

Published: April 9, 2020

Reference information: /CI Insight. 2020;5(7):e136041.

https://doi.org/10.1172/jci.

insight.136041.

\section{Introduction}

Achromatopsia (ACHM; also known as rod monochromatism) is an autosomal recessive form of cone dysfunction that presents in infancy. It is characterized by reduction of visual acuity, photophobia, nystagmus, and poor color vision $(1,2)$. To date, 6 genes have been found to cause ACHM: cyclic nucleotide-gated cation channel B3 (CNGB3) (3), cyclic nucleotide-gated cation channel A3 (CNGA3) (4), guanine nucleotide-binding $\mathrm{G}(\mathrm{t})$ subunit A2 (GNAT2) (5), cone cyclic GMP-specific 3',5' phosphodiesterase 6A (PDE6C) (6), cone cyclic GMP-specific phosphodiesterase G $(P D E 6 H)(7)$, and activating transcription factor 6 (ATF6) (8). ATF6 is the most recent of these disease genes to be identified (8-12), and, in contrast to other ACHM disease genes, it is not involved in the cone phototransduction pathway (13).

ATF6 encodes a glycosylated 670-amino acid type 2 ER transmembrane protein $(14,15)$. The ATF6 protein consists of a luminal ER stress-sensing domain coupled across the ER membrane to a cytosolic basic leucine zipper (bZIP) domain transcription factor. It is an important regulator of the unfolded protein response (UPR), an intracellular signal transduction mechanism that prevents ER stress and ensures ER homeostasis (16). In response to ER stress, monomeric ATF6 protein migrates from the ER to the Golgi apparatus where it 
is cleaved in the transmembrane domain by Golgi-resident site 1 and site 2 proteases, releasing its cytosolic bZIP transcription factor domain $(17,18)$. The liberated ATF6 bZIP transcription factor domain then enters the nucleus and upregulates target genes that include ER chaperones, such as BiP/Grp78 and protein-folding enzymes $(14,19,20)$. Thus, ATF6 signaling helps cells survive ER stress by increasing the cell's protein-folding capability.

To date, 11 different ATF6 disease alleles have been identified in patients with ACHM (8, 9, 11, 12, $21)$, containing missense, nonsense, and indel mutations or splice-site changes. In this paper, we identified and characterized 2 additional multiexon-spanning ATF6 disease alleles in patients with ACHM. Consistent with prior analyses of other ATF6 ACHM alleles, we found that recombinant ATF6 proteins with these large deletions show severely impaired transcriptional activity. The data support the hypothesis that intact ATF6 transcription factor function is necessary for cone photoreceptor function and survival $(8,21)$. Interestingly, we found, using the RNAscope assay, that ATF6 was expressed in cones and throughout the retinal layers. Thus, defects in ATF6 may also have an important role in visual processing of the retina.

\section{Results}

Multiexon deletions in ATF6 found in patients with ACHM. Eleven ATF6 photoreceptor disease alleles have been previously identified in patients with ACHM or cone-rod dystrophy; these alleles contain single-nucleotide changes (i.e., missense, nonsense, and splice site mutations), small deletions, or duplications that disrupt ATF6 production or function (Table 1) $(8,9,11,12,21)$. In the current study, we identified 2 ATF6 mutations that delete large fragments of the ATF6 gene, leading to loss of multiple exons (Table 1).

In 2 siblings from family A, we identified a homozygous deletion, c.909+1_1720-1del, that leads to theloss of exons 8-14 (Table 1 and Figure 1A). The exact breakpoint is defined as NG_029773.1:g.58488_115797delinsAGAGCTC; NG_029773:1(ATF6_v001):c.1008_1719+13728delinsAGAGCTC. Segregation in both parents using breakpoint PCR analysis showed that both parents are heterozygous carriers of the deletion (Figure 1A). In another patient from family B, we studied a heterozygous deletion, c.82+1_248-1del, that leads to the loss of exons 2 and 3 (Table 1 and Figure 1A) (10). The patient has a second heterozygous mutation, c.970C>T;p.Arg324Cys, previously reported in other patients with ACHM (8). The parents subsequently underwent genetic testing and were found to be heterozygous carriers of either the previously characterized c.970C > T, (p.Arg324Cys) ATF6 allele or the c.82+1_248-1del ATF6 allele, respectively (Figure 1A). The parents from both families A and B had no visual defects. Furthermore, parents reported no consanguinity. At early infancy, all patients presented reduced visual acuity, nystagmus, and photophobia.

Both novel mutations (i.e., exons 8-14 deletion and exons 2-3 deletion) in ATF6 affect domains of the ATF6 transcriptional activity (Figure 1B). If the deletion flanking exons are spliced directly onto each other, both deletions in the mRNA are in-frame. The exon 8-14 deletion removed 270 amino acid residues, including the bZIP domain, the transmembrane domain, and most of the luminal domain of ATF6 (Figure 1B). When exons 2 and 3 were deleted, 55 amino acid residues were removed, leading to removal of part of the transcriptional activator domain of ATF6 (Figure 1B).

The patient with the c.82+1_248-1del ATF6 allele also carries a SNP in ATF6 (rs1058405) that introduces a Met67Val amino acid change in the protein (Figure 1B, M67V). Methionine and valine are aliphatic, nonreactive amino acids with similar molecular structures, suggesting that this conservative substitution is neither pathogenic to the ATF6 conformation nor detrimental to its function. Furthermore, this variant is widespread in the general population, with an allele frequency of $2.31 \times 10^{-1}$ (https://gnomad.broadin stitute.org/gene/ENSG00000118217?dataset=gnomad_r2_1), making it unlikely to be a disease-causing variant. For comparison, the allele frequency of the pathogenic c.970C >T;(p.Arg324Cys) ATF6 allele is $2.13 \times 10^{-5}$ (https://gnomad.broadinstitute.org/gene/ENSG00000118217?dataset=gnomad_r2_1). However, prior biochemical experiments reported functional differences for the M67V ATF6 variant (22).

Functional analysis of ATF6 alleles with large multiexon deletions. To determine how deletion of exons 8-14 or exons 2-3 in ATF6 affects ATF6 function, and to experimentally determine whether the Met67Val ATF6 variant causes pathogenic consequences for ATF6 function, we transfected HEK293 cells with FLAGtagged cytosolic domains of WT-ATF6, the Met67Val variant of ATF6 (ATF6[M67V]), an ACHM-linked N267* (ATF6[N267*]) (8, 21), ATF6[D28_T82del], and ATF6[I304-R573del] (Figure 2A). The WT-ATF6 cytosolic domain served as the positive control and $\mathrm{N} 267^{*}$, a previously identified ACHM-linked mutation that does not possess any ATF6 transcriptional activity, served as a negative control (21).

To determine the transcriptional activity on the ATF6 variants, we first investigated the protein expression levels of BiP/GRP78 (one of the most highly induced transcriptional targets of ATF6, refs. 
Table 1. Summary of identified ATF6 disease alleles

\begin{tabular}{|c|c|c|c|}
\hline Nucleotide change & Effect & Protein & Reference \\
\hline c. $82+5 G>T$ & Splicing defect & p.D28Gfs*36 & 8 \\
\hline c.353delC & Truncation & p.P118Lfs*31 & 8 \\
\hline c.355_356dupG & Truncation & p.E119Gfs*8 & 9 \\
\hline c.797dupC & Truncation & p.N267* & 8 \\
\hline c. $970 C>T$ & Point mutation & p.R324C & 8 \\
\hline c.1110dupA & Truncation & p.V371Sfs*3 & 8 \\
\hline c. $1126 C>T$ & Truncation & p.R376* & 12 \\
\hline c. $1187+5 G>C$ & Splicing defect & p.N366Hfs*12 & 8 \\
\hline c. $1533+1 G>C$ & Splicing defect & $\begin{array}{l}\text { p.G512Lfs*39 } \\
\text { p.L479Vfs*11 }\end{array}$ & $\begin{array}{l}8 \\
8\end{array}$ \\
\hline c.1691A>G & Point mutation & p.D564C & 11 \\
\hline с.1699T>A & Point mutation & p.Y567N & 8 \\
\hline c.909+1_1720-1del & Exon 8-14 deletion & p.I304_R573del & Current study \\
\hline c.82+1_248-1del & Exon 2-3 deletion & p.D28_T82del & Current study \\
\hline
\end{tabular}

Two new multiexon deletion alleles of ATF6 were identified in patients. The nucleotide changes and molecular and protein effects of the nucleotide changes of the 2 new multiexon deletion alleles (red) and previously identified ATF6 alleles are listed. Variants are presented with respect to ATF6 (RefSeq, NM_007348.3).

14, 23) by immunoblot analysis using antibodies against BiP/GRP78. The BiP/GRP78-immunoreactive band was present in all groups (Figure 2A). However, the intensity of the BiP/GRP78-immunoreactive band was weak in all the groups except for WT-ATF6 and ATF6[M67V]. Next, we performed qRT-PCR analysis on these transfected samples and found that ATF6 variants encoding the ACHM-linked N267* ATF6[N267]*, ref. 8)], ATF6[D28_T82del], and ATF6[I304-R573del] failed to transcriptionally induce expression of ATF6 target genes BiP/GRP78, HerpUD1, and Chop (24-26) (Figure 2B). By contrast, the mRNA levels of BiP/GRP78, HerpUD1, and Chop were induced in WT-ATF6 and ATF6[M67V]. We did not observe changes in $E R d j 4$ (a downstream target gene induced by the IRE1 signaling pathway) mRNA among the experimental groups

To further characterize the transcriptional activity of these ATF6 variants, we examined a 17-gene panel of ATF6 transcriptional targets, previously defined by RNA-Seq of HEK293 cells expressing intact ATF6 transcriptional activator fragment (27). Strong induction of ATF6 target genes by RNA-Seq was found in transfected HEK293 cells with WT-ATF6 or the Met67Val ATF6 variant (ATF6[M67V]) (Figure 3, bottom 2 rows). Expression of ATF6[N267*], ATF6[D28_T82del], and ATF6[I304-R573del] revealed no induction of these ATF6 targets (Figure 3, top 3 rows). Our qRT-PCR and RNA-Seq analyses reveal that ATF6 transcriptional activity was lost in ATF6[N267*], ATF6[D28_T82del], and ATF6[I304-R573del] mutations, showing similarities to previously characterized ACHM-associated ATF6 variants (8).

Clinical findings in the patient with exons 2 and 3 of the ATF6 gene deleted in this mutation. The patient with the deletion of exons 2-3 in the ATF6 allele is a 7-year-old boy, who, starting in infancy, presents nystagmus, impaired visual acuity, and photophobia. Fundus examination demonstrated crowded optic discs in both eyes and mild macular atrophy (Figure 4A). Fundus autofluorescence imaging showed mild foveal hyperautofluorescence (Figure 4B). Spectral domain-optical coherence tomography showed severe foveal hypoplasia in the right (Figure 4C, top) and left (Figure 4C, bottom) eyes and focal disruption in the inner segment ellipsoid layer at the site of the presumed foveal pit (Figure 4C, arrowheads). In addition, the photopic full-field (light-adapted 3.0) electroretinogram (ERG) recordings showed defective (barely detectable) amplitudes for the a-wave and b-wave responses (Table 2) in the patient with the deletion of exons $2-3$ in ATF6. These findings are consistent with prior imaging studies of ATF6-associated patients with ACHM (10).

Expression of $A T F 6$ and $A T F 6 B m R N A$ in human retina. ATF6 is a member of the bZIP transcription factors and is encoded as 2 isoforms, ATF6 and ATF6B (8). Other ACHM disease genes are specifically expressed in cones and are all functional components of the phototransduction cascade. To determine the localization and expression of ATF6 and ATF6B in the human retina, and especially in the cones, we performed triple-labeling experiments using RNA oligo probes specific for the mRNA of human retinal cone arrestin-3 (Arr3), ATF6, and $A T F 6 B$ in vertical cross sections of the human adult retina. Human retinal 
A

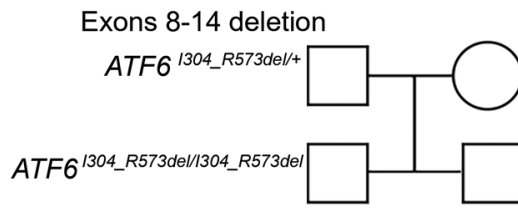

ATF6 1304_R573del/+

ATF6 1304_R573del/1304_R573del

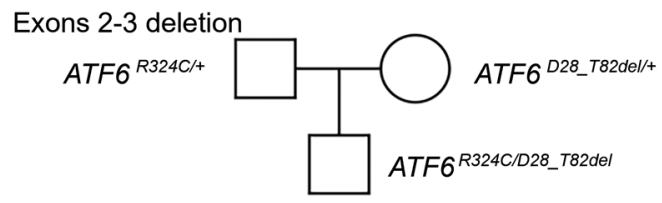

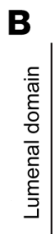

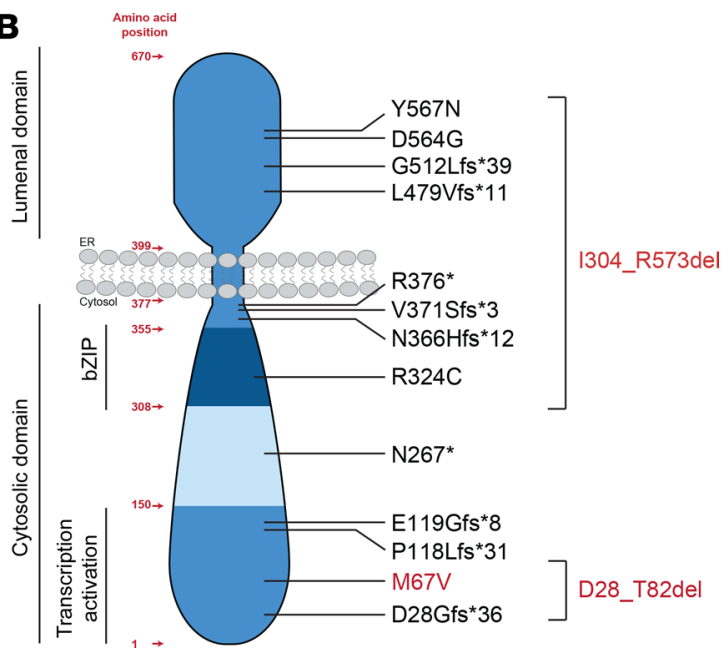

Figure 1. Pedigrees and topography of disease-causing mutations identified in the patients. (A) Pedigree drawings of patients with deletions of exons 8-14 and exons 2-3 in ATF6. (B) The amino acid organization, protein topology, and functional domains of ATF6 are shown, and the positions of the ATF6 variants are mapped onto the schematic drawing. Variants tested in this study are shown in red.

Arr3 is a cone specific marker (28-30). Figure 5 shows an example of a vertical section of the midperiphery of the human retina triple labeled with $A r r 3, A T F 6$, and $A T F 6 B$. In situ hybridization results showed that the mRNA probe for $A r r 3$ is found in the cell bodies and outer segments of cones; furthermore, mRNA probes for $A T F 6$ and $A T F 6 B$ are observed in all retinal layers. In a merged figure, $A T F 6$ and $A T F 6 B$ mRNA puncta were localized within the cell bodies that are labeled with Arr3 mRNA in the outer nuclear layer. In addition, $A T F 6$ and $A T F 6 B$ puncta were localized in the outer plexiform layer, inner nuclear layer, inner plexiform layer, and ganglion cell layer. Our data show that ATF6 was not only expressed in cones, but also widely expressed in all the retinal layers.

\section{Discussion}

ATF6 mutations have previously been found in patients with ACHM or cone-rod dystrophy and those patients carried single-nucleotide changes (i.e., missense, nonsense, and splice site mutations), small deletions, and small duplications of the gene $(8,9,11,12)$. These mutations lead to loss of ATF6 function by damaging the amino-terminal transcriptional activator/bZIP DNA-binding domain of ATF6 or impairing the carboxyl-terminal ER to Golgi intracellular protein trafficking domain of ATF6 (8, 21). Here, we study two new larger genomic deletions of ATF6 in 3 patients with ACHM that lead to multiexon deletions within ATF6. The deletion c.909+1_1720-1del in the ATF6 gene identified in the affected siblings removes the in-frame bZip, transmembrane, and luminal domains of ATF6 (exon 8-14 deletion, p.I304_R573del, Figure 1B). The deletion c.82+1_248-1del in the $A T F 6$ gene identified in the affected single patient removes an in-frame part of the transcriptional activation domain (exons 2-3 deletion, p.D28_T82del, Figure 1B). The effect on ATF6 function in cells expressing these disease-causing variants was tested with recombinant ATF6[N267*] (8, 21), ATF6[D28_T82del], and ATF6[I304-R573del] ATF6 proteins. Our transcriptomic analyses demonstrate that the ACHM-linked N267* and ATF6 variants, encoding p.D28_T82del and p.I304-R573del, show severely reduced induction of ATF6 transcriptional target genes compared with the WT-ATF6 and ATF6[M67V] by qRT-PCR and by RNA-Seq. These findings demonstrate that both multiexon deletion $A T F 6$ variants completely lose transcriptional activator function. These findings further underscore that all ACHM-linked ATF6 variants identified to date impair transcriptional activity.

The 2 isoforms of ATF6 share significantly conserved bZIP domains and DNA-binding domains; however, the ATF6 isoform is better studied than ATF6B biochemically and physiologically (31-35). In the current study, we examined the expression of both ATF6 and ATF6B mRNA by in situ hybridization in the human retina and RNAscope assay. The RNAscope assay is based on the branched DNA method described in previous studies (36-39). RNAscope in situ hybridization decreases nonspecific hybridization and increases signal amplification by using a $\mathrm{Z}$ probe, which has $25 \mathrm{bp}$ of oligonucleotides that are complementary to a region of the $A T F 6$ and $A T F 6 B$ mRNA. We observed $A T F 6$ and $A T F 6 B$ mRNA expression 
A

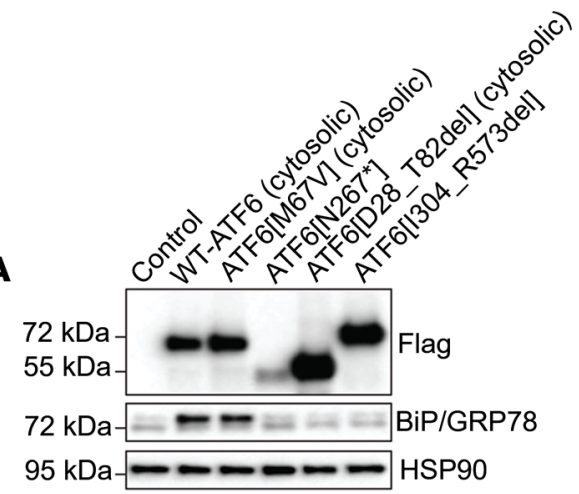

B
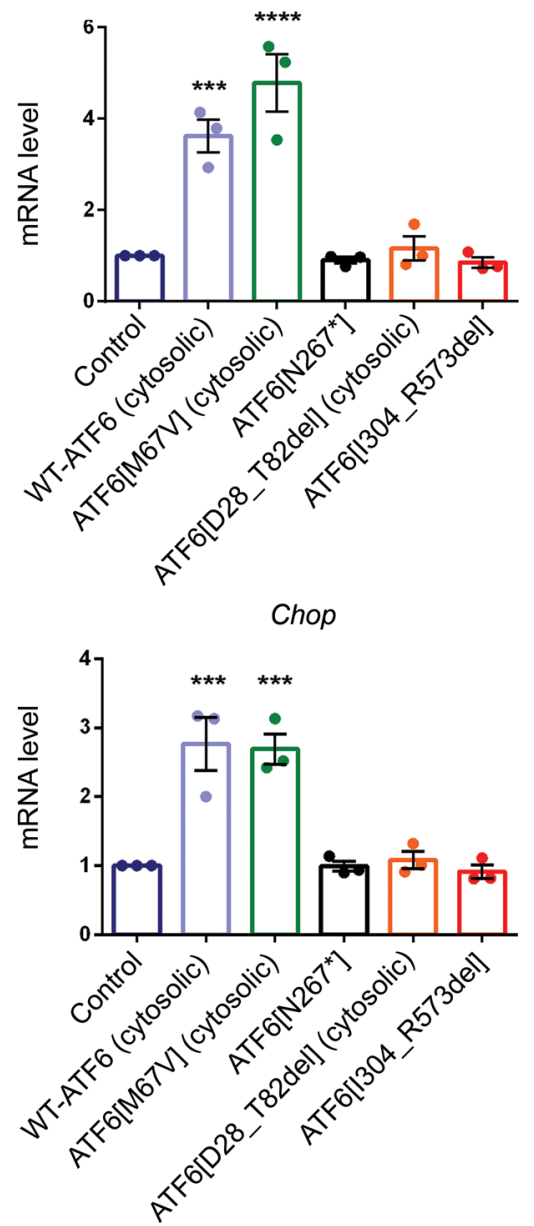

HerpUD1

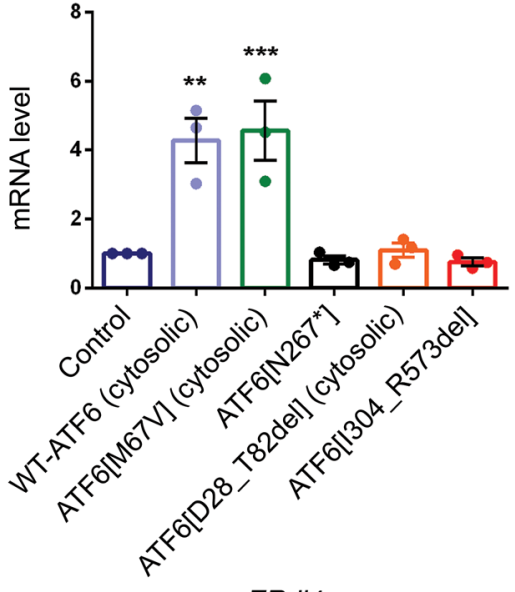

ERdj4

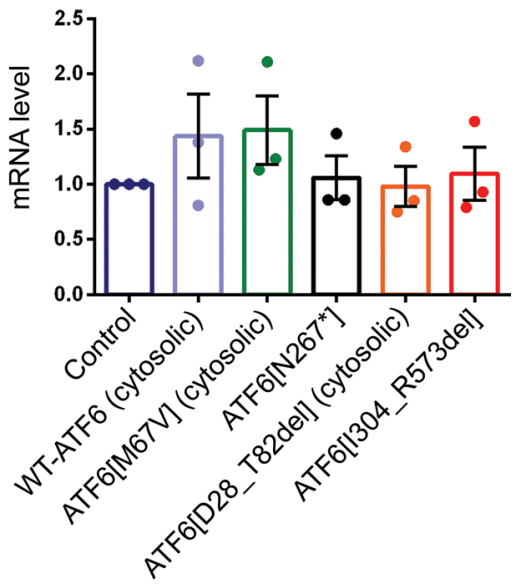

Figure 2. Functional characterization of the ATF6 multiexon deletion variants. (A) HEK293 cells were transfected with plasmids encoding the cytosolic transcriptional activator domains of WT-ATF6, the M67V ATF6 variant (ATF6[M67V]), the ACHM-linked N267* ATF6 variant (ATF6[N267*]), the variant with deletion of exons 2-3 in ATF6 (ATF6[D28_T82del]), or the variant with deletion of exons 8-14 in ATF6 (ATF6[I304_R573del]). Cell lysates were immunoblotted for recombinant ATF6 protein expression (anti-FLAG), BiP/Grp78 protein, and HSP9O (loading control). (See complete unedited blots in the supplemental material.) (B) The mRNA levels of ATF6 downstream target genes (BIP, HerpUD1, and Chop) and an XBP1 transcriptional target gene (ERdj4) were measured by real-time quantitative PCR and normalized to mRNA levels in untransfected control samples. Data are shown as mean \pm SEM; 3 sets of independent experiments were performed. Data in B were analyzed by 1-way ANOVA and showed significance at ${ }^{* *} P<0.01$; ${ }^{* *} P<0.001$; ${ }^{* * *} P<0.0001$ 


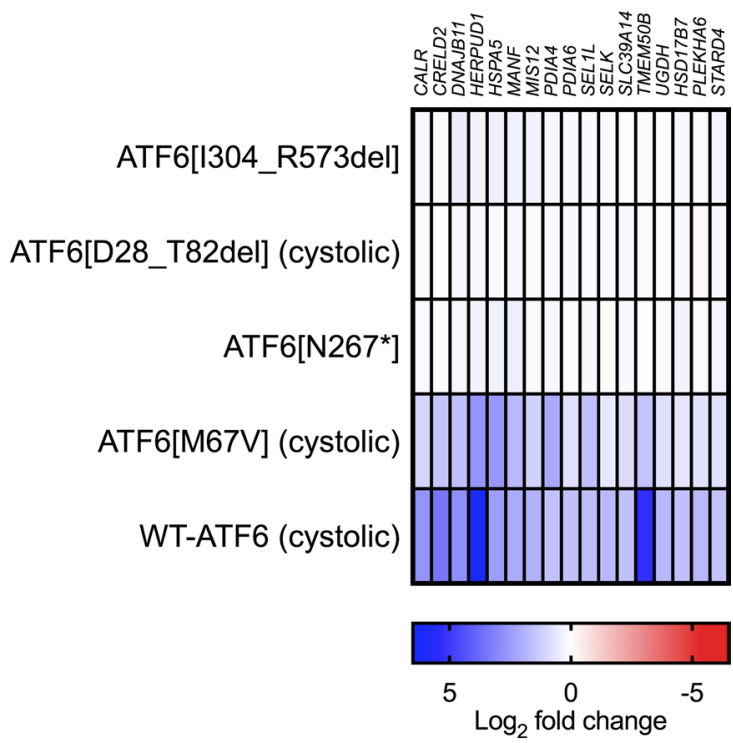

Figure 3. The RNA-Seq analysis. The heatmap shows the $\log _{2}$ fold changes of 17 ATF6 target genes in the RNA-Seq analysis of HEK293 cells transfected with WT-ATF6, ATF6[M67V], ATF6[N267*], ATF6[D27_T82del], and ATF6[[1304_R573del].

on the cone photoreceptors and in all the retinal layers of the human retina by RNAscope in situ hybridization. The mRNA expression of $A T F 6$ and $A T F 6 B$ described in the current study is consistent with previously performed immunohistochemistry studies in the mouse retina, which also show ATF6 expression in all the retinal layers $(9,12)$. Furthermore, several in vitro studies show that ATF6 expression in inner retinal cells (e.g., retinal ganglion cells) is induced after ER stress (40-42). Together with our current results, these findings suggest that ATF6 plays an essential role in regulating ER stress in all retinal neurons. The reasons why loss of ATF6 preferentially causes a cone defect are unclear. One possibility is that other retinal cells have additional mechanisms for dealing with ER stress not found in cones. Another possibility is that cones undergo unique forms of ER stress that specifically require ATF6 function.

In recent years, structurally distinct small molecules have been identified that can activate or inhibit ATF6 or other parallel signal transduction pathways of the UPR to help cells tolerate ER stress (27, 43-46). Our functional studies support that ATF6 mutations linked to retinal development disease arise from loss of ATF6 transcriptional activity, and, in turn, this is caused by defects at distinct steps of ATF6 activation $(8,21)$. Based on these findings, ATF6-activating compounds may be helpful to correct the vision loss disease arising from ATF6 mutations in patients. In particular, in patients carrying loss-of-function ATF6 alleles arising from ER retention of the ATF6 protein, the activator of ATF6 (N-(2-hydroxy-5-methylphenyl)-3-phenylpropanamide [AA147]) (27), may be helpful to restore activity through promotion of ATF6 ER-to-Golgi trafficking. Alternatively, small molecules that induce UPR transcription factors with similar downstream transcriptional programs to ATF6, such as XBP1, may be helpful to compensate for loss of ATF6 (27, 47).

\section{Methods}

Patients. Patients were clinically evaluated at 2 centers (Institute de la Vision, Paris, France, and Medical College of Wisconsin). For RNAscope in situ hybridization, adult human eye samples were collected with appropriate consent from the San Diego Eye bank (product code V0180).

Clinical evaluation. Spectral-domain optical coherence tomography, fundus, and autofluorescence (Heidelberg Engineering) imaging were performed using standard methods using the Heidelberg Spectralis (8). Light-adaptation thresholds full-field ERG was performed on all patients using the International Society for Clinical Electrophysiology of Vision standard protocols using JET disposable contact electrodes (48).

Genetic analysis and screening of ATF6. The patient with the deletion of exons 2-3 in ATF6 and his father underwent genetic testing using Retinal Dystrophy Panel Plus (Blueprint Genetics), which covers 266 genes known to cause inherited retinal diseases discovered by high-throughput sequencing and deletion/duplication analysis. The siblings with the deletion of exons 8-14 in ATF6 underwent genetic testing at the Institute of Ophthalmology, Tuebingen, Germany, by a step-wise procedure analyzing the genes known to be associated with ACHM by PCR and Sanger sequencing as previously described (8). The exact breakpoint was 

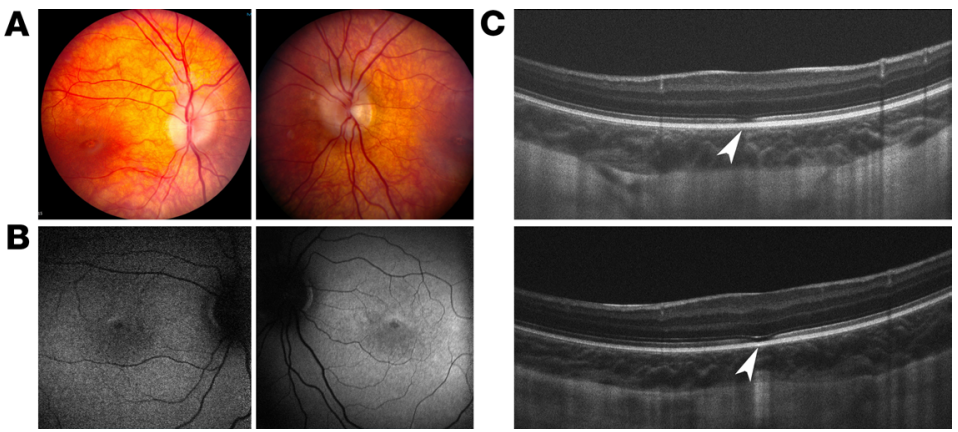

Figure 4. Clinical findings for the patient with deletion of exons $\mathbf{2}$ and $\mathbf{3}$ in ATF6. Fundus images of right and left eyes of the patient showed crowded optic discs and mild macular atrophy (A). Autofluorescence images of right and left eyes show mild hyperautofluorescence in fovea (B). Spectral domain OCT images showed foveal hypoplasia in right (top) and left (bottom) eyes (C). Inner segments and outer segments were disrupted at the fovea region (arrowheads).

defined by breakpoint PCR and Sanger sequencing.

Biochemical assays. The WT-ATF6(aa 1-373), ATF6[Met67Val(aa 1-373)], ATF6[N267*], and ATF6[D28_T82del] expression plasmids were generated by site-directed mutagenesis using the QuikChange II Site-Directed Mutagenesis Kit (Agilent Technologies Inc.) in the coding region of FLAGtagged-ATF6(aa 373) pcDNA3.1 plasmid. The ATF6[1304-R573del] expression plasmid was generated in the coding region of FLAG-tagged-ATF6(full-length) pcDNA3.1 as previously described (21). ATF6 plasmids were transiently transfected into HEK293 cells, using Lipofectamine 2000 (Invitrogen). HEK293 cells expressing WT or mutant ATF6 were lysed in SDS lysis buffer (2\% SDS in PBS) and phosphatase inhibitor (Thermo Scientific). Protein concentrations of the total cell lysates were determined by BCA protein assay (Pierce). Equal amounts of protein were applied onto 4\%-15\% Mini-PROTEAN TGX precast gels (Bio-Rad) and analyzed by immunoblot. The following antibodies and dilutions were used: anti-FLAG at 1:5,000 (catalog A8592, MilliporeSigma), anti-BiP/GRP78 at 1:1,000 (catalog GTX113340, GeneTex Inc.), and anti-HSP90 at 1:5,000 (catalog GTX101448, GeneTex Inc.). After overnight incubation with primary antibody, membranes were washed in TBS with $0.1 \%$ Tween-20, followed by incubation of a horseradish peroxidase-coupled secondary antibody (catalog 7074, Cell Signaling). Immunoreactivity was detected using the Super-Signal West chemiluminescent substrate (Pierce).

qRT-PCR analysis. HEK293 cells expressing WT and mutant ATF6 were lysed, and total RNA was collected using the RNeasy mini kit, according to manufacturer's instructions (Qiagen). The mRNA was reverse-transcribed using the iScript cDNA Synthesis Kit (Bio-Rad). For quantitative PCR analysis, cDNA was used as template in SYBR Green qPCR Supermix (Bio-Rad). Primers used include human BIP/GRP78 (5'-GCCTGTATTTCTAGACCTGCC-3' and 5'-TTCATCTTGCCAGCCAGTTG-3'), human HERPUD1 (5'-AACGGCATGTTTTGCATCTG-3' and 5'-GGGGAAGAAAGGTTCCGAAG-3'), human CHOP (5'-ACCAAGGGAGAACCAGGAAACG-3' and 5'-TCACCATTCG-

Table 2. ERG recordings for the patient with deletion of exons 2-3 in ATF6

\begin{tabular}{|c|c|c|c|c|c|c|}
\hline & \multicolumn{2}{|c|}{ OD } & \multicolumn{2}{|c|}{ OS } & \multicolumn{2}{|c|}{ Normal } \\
\hline & a-wave & b-wave & a-wave & b-wave & a-wave & b-wave \\
\hline \multicolumn{7}{|c|}{ Light-adapted 3.0 ERG } \\
\hline Amplitude (mV) & -34.92 & 24.95 & -33.76 & 25.2 & $-64.17 \pm 38.03$ & $178.8 \pm 116.9$ \\
\hline Implicit times (ms) & 20 & 36 & 14 & 35 & $16.33 \pm 1.56$ & $27.5 \pm 3.13$ \\
\hline \multicolumn{7}{|c|}{$\begin{array}{l}\text { International Society for Clinical Electrophysiology of Vision standard ERG recordings for the patient with deletion of } \\
\text { exons } 2-3 \text { and a healthy control individual. The full-field flash strength (in cd·s } / \mathrm{m}^{2} \text { ) after light adaptation (photopic } \\
\text { response) for the right eye (OD) and left eye (OS). The amplitude for a-wave and b-wave was not within the normal } \\
\text { ranges under photopic condition in the patient. }\end{array}$} \\
\hline
\end{tabular}




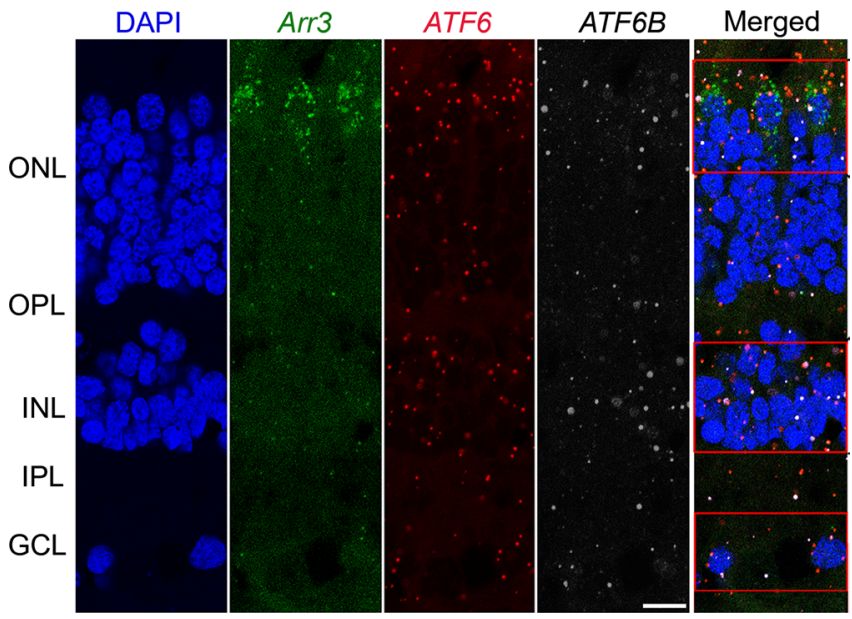

Figure 5. Confocal micrographs of vertical sections of RNAscope in situ hybridization. Vertical section triple labeled with Arr3 (green), ATF6 (red), and ATF6B (white) mRNA in normal human retina. Nuclei were labeled with DAPI (blue) to identify the nuclear layers of the retina. The mRNA probe for Arr3 was found in the cell bodies and inner segments of cones, and the mRNA probes for ATF6 (red) and ATF6B (white) were observed in all the retinal layers. In the merged image, ATF6 and ATF6B mRNA puncta were localized within the cell bodies that are labeled with Arr3 in the outer nuclear layer (ONL). Three sets of independent experiments were performed $(n=2$ retinas). Expression of ATF6 and ATF6B puncta was localized in the ONL, outer plexiform layer (OPL), inner nuclear layer (INL), inner plexiform layer (IPL), and ganglion cell layer (GCL). Scale bar: $10 \mu \mathrm{m}$.

GTCAATCAGAGC-3'), human ERdj4 (5'-GGAAGGAGGAGCGCTAGGTC-3' and 5'-ATCCTGCACCCTCCGACTAC-3'), human ATF6 (5'-GCTTTACATTCCTCCACCTCCTTG-3' and 5'-ATTTGAGCCCTGTTCCAGAGCAC-3'), and human total XBP1 (5'-GCAAGCGACAGCGCCT-3' and 5'-TTTTCAGTTTCCTCCTCAGCG-3'). RPL19 mRNA levels (human RPL19, 5'-ATGTATCACAGCCTGTACCTG-3' and 5'-TTCTTGGTCTCTTCCTCCTTG-3') served as internal normalization standards. The qPCR condition was $95^{\circ} \mathrm{C}$ for 5 minutes, $95^{\circ} \mathrm{C}$ for 10 seconds, $60^{\circ} \mathrm{C}$ for 10 seconds, and $72^{\circ} \mathrm{C}$ for 10 seconds, with 40 cycles of amplification.

RNA-Seq analysis. HEK293 cells expressing WT or mutant ATF6 were collected for RNA-Seq analysis. Cells were lysed and total RNA was collected using a Qiagen kit (RNeasy Mini Kit) in line with the manufacturer's recommendations and previous study (49). Three sets of independent experiments were performed. Briefly, conventional RNA-seq was conducted via BGI Americas on the BGI Proprietary platform, providing single-end 50-bp reads at 20 million reads per sample. Alignment of sequencing data was done using DNAstar Lasergene SeqManPro to the GRCh37.p13 human genome reference assembly; assembly data were imported into ArrayStar 12.2 with QSeq (DNAStar Inc.) to quantify the gene expression levels and normalized to reads per kilobase per million (RPKM). Differential expression analysis and statistical significance calculations between different conditions were assessed using "DESeq" in R, compared with WT controls, with a standard negative binomial fit for the RPKM data. Heatmaps were generated using the $\log _{2}$ fold change data from this normalized differential expression analysis for genes of interest.

RNAscope assay. In situ RNA hybridization was performed using the RNAscope assay (Advanced Cell Diagnostics) according to the manufacturer's recommendations and previous study (38). Briefly, the anterior segment and crystalline lens were removed, and the eye cups were fixed with $4 \%$ paraformaldehyde and processed for cryostat sections $(20 \mu \mathrm{m})$. The vertical sections were subjected to RNAscope Multiplex Fluorescent Assay. The sections were incubated with pretreatment 2 (RNAscope Target Retrieval Reagents, Advanced Cell Diagnostics) for 30 minutes at $40^{\circ} \mathrm{C}$ in a HybEZ oven (HybEZ Hybridization System, Advanced Cell Diagnostics), followed by pretreatment 3 (RNAscope ProteaseIII, Advanced Cell Diagnostics) containing protease for 30 minutes at $40^{\circ} \mathrm{C}$. After pretreatment 3 , the sections were washed with $5 \times$ deionized water and incubated with the prewarmed mRNA target probes (ATF6-C1, 555291; Arr3-C2, 486461; or ATF6B-C3, 555301 [Advanced Cell Diagnostics]) for 2 hours at $40^{\circ} \mathrm{C}$ in the HybEZ oven. No-probe controls were performed in parallel (Supplemental Figure 1; supplemental material available online with this article; https://doi.org/10.1172/jci.insight.136041DS1). The sections were processed in wash buffer for 5 minutes and then incubated with Amp 1 for 30 minutes at $40^{\circ} \mathrm{C}$ and washed with washing buffer for 5 minutes. The procedure was repeated for Amp2, Amp3, HRP-C1 (Cy3), HRP-C2 (FITC), and HRP-C3 (Cy5). After washing with washing buffer for 5 minutes, the sections were coverslipped with Vectashield-DAPI mounting medium (Vector Laboratories). The images of sections were saved and processed with the Leica application suitex software under a Leica SP8 confocal microscopy and then were adjusted using Adobe Photoshop 7.0. Photoshop adjustments were carried out equally across sections. According to Advanced Cell Diagnostics, each mRNA molecule hybridized to a probe appears as separate small puncta. 
Statistics. Data are expressed as mean \pm SEM. Comparisons of multiple groups were performed using 1-way ANOVA. All in vitro studies were done with at least 3 sets of independent experiments. GraphPad Prism was used for all analyses. A $P$ value of less than 0.05 was considered significant.

Study approval. This study was approved by the Institutional Review Boards of UCSD, the Medical College of Wisconsin, the University Tuebingen, and Columbia University and conducted in accordance with the tenets of the Declaration of Helsinki and NIH guidelines. Patients and parents of the patients provided informed consent for the study.

\section{Author contributions}

EJL, WCJC, and JHL conceived and designed the study. EJL, WCJC, HK, CXB, JMDG, LC, DSK, KK, and RLW performed the biochemical, transcriptomic, and RNAscope experiments. DLC, SHT, RRM, SRL, IA, ATM, and JC performed the clinical imaging. BB and SK performed the genetic analyses. All authors contributed to data analyses. EJL and JHL wrote the manuscript, and all authors contributed to manuscript editing.

\section{Acknowledgments}

Research results reported in this publication were supported by NIH awards R01EY017607, AG046496, U54OD020351, R01EY018213, and R01EY027335; the California Institute for Regenerative Medicine DISC2-10973 award; and VA Merit Award I01BX002284.

Address correspondence to: Jonathan Lin, School of Medicine, Stanford University, 300 Pasteur Drive L235, Palo Alto, California 94304, USA. Phone: 650.497.3018; Email: jlinn@stanford.edu.

1. Aboshiha J, Dubis AM, Carroll J, Hardcastle AJ, Michaelides M. The cone dysfunction syndromes. Br J Ophthalmol. 2016;100(1):115-121.

2. Hirji N, Aboshiha J, Georgiou M, Bainbridge J, Michaelides M. Achromatopsia: clinical features, molecular genetics, animal models and therapeutic options. Ophthalmic Genet. 2018;39(2):149-157.

3. Kohl S, et al. Mutations in the CNGB3 gene encoding the beta-subunit of the cone photoreceptor cGMP-gated channel are responsible for achromatopsia (ACHM3) linked to chromosome 8q21. Hum Mol Genet. 2000;9(14):2107-2116.

4. Kohl S, et al. Total colourblindness is caused by mutations in the gene encoding the alpha-subunit of the cone photoreceptor cGMP-gated cation channel. Nat Genet. 1998;19(3):257-259.

5. Kohl S, et al. Mutations in the cone photoreceptor G-protein alpha-subunit gene GNAT2 in patients with achromatopsia. Am $J$ Hum Genet. 2002;71(2):422-425.

6. Chang B, et al. A homologous genetic basis of the murine cpfl1 mutant and human achromatopsia linked to mutations in the PDE6C gene. Proc Natl Acad Sci USA. 2009;106(46):19581-19586.

7. Kohl S, et al. A nonsense mutation in PDE6H causes autosomal-recessive incomplete achromatopsia. Am J Hum Genet. 2012;91(3):527-532.

8. Kohl S, et al. Mutations in the unfolded protein response regulator ATF6 cause the cone dysfunction disorder achromatopsia. Nat Genet. 2015;47(7):757-765.

9. Ansar M, et al. Mutation of ATF6 causes autosomal recessive achromatopsia. Hum Genet. 2015;134(9):941-950

10. Mastey RR, et al. Characterization of retinal structure in ATF6-associated achromatopsia. Invest Ophthalmol Vis Sci. 2019;60(7):2631-2640.

11. Skorczyk-Werner A, et al. Autosomal recessive cone-rod dystrophy can be caused by mutations in the ATF6 gene. Eur J Hum Genet. 2017;25(11):1210-1216.

12. Xu M, et al. ATF6 is mutated in early onset photoreceptor degeneration with macular involvement. Invest Ophthalmol Vis Sci. 2015;56(6):3889-3895.

13. Hamel CP. Cone rod dystrophies. Orphanet J Rare Dis. 2007;2:7.

14. Haze K, Yoshida H, Yanagi H, Yura T, Mori K. Mammalian transcription factor ATF6 is synthesized as a transmembrane protein and activated by proteolysis in response to endoplasmic reticulum stress. Mol Biol Cell. 1999;10(11):3787-3799.

15. Hong M, et al. Underglycosylation of ATF6 as a novel sensing mechanism for activation of the unfolded protein response. $J$ Biol Chem. 2004;279(12):11354-11363.

16. Walter P, Ron D. The unfolded protein response: from stress pathway to homeostatic regulation. Science. 2011;334(6059):1081-1086

17. Nadanaka S, Yoshida H, Mori K. Reduction of disulfide bridges in the lumenal domain of ATF6 in response to glucose starvation. Cell Struct Funct. 2006;31(2):127-134.

18. Okada A, et al. OsTGAP1, a bZIP transcription factor, coordinately regulates the inductive production of diterpenoid phytoalexins in rice. J Biol Chem. 2009;284(39):26510-26518.

19. Shen J, Snapp EL, Lippincott-Schwartz J, Prywes R. Stable binding of ATF6 to BiP in the endoplasmic reticulum stress response. Mol Cell Biol. 2005;25(3):921-932.

20. Wang Y, Shen J, Arenzana N, Tirasophon W, Kaufman RJ, Prywes R. Activation of ATF6 and an ATF6 DNA binding site by the endoplasmic reticulum stress response. J Biol Chem. 2000;275(35):27013-27020.

21. Chiang WC, et al. Achromatopsia mutations target sequential steps of ATF6 activation. Proc Natl Acad Sci USA. 
2017;114(2):400-405.

22. Meex SJ, et al. The ATF6-Met[67]Val substitution is associated with increased plasma cholesterol levels. Arterioscler Thromb Vasc Biol. 2009;29(9):1322-1327.

23. Yoshida H, Haze K, Yanagi H, Yura T, Mori K. Identification of the cis-acting endoplasmic reticulum stress response element responsible for transcriptional induction of mammalian glucose-regulated proteins. Involvement of basic leucine zipper transcription factors. J Biol Chem. 1998;273(50):33741-33749.

24. Oyadomari S, Mori M. Roles of CHOP/GADD153 in endoplasmic reticulum stress. Cell Death Differ. 2004;11(4):381-389.

25. Yoshida H, et al. ATF6 activated by proteolysis binds in the presence of NF-Y (CBF) directly to the cis-acting element responsible for the mammalian unfolded protein response. Mol Cell Biol. 2000;20(18):6755-6767.

26. Bommiasamy H, et al. ATF6alpha induces XBP1-independent expansion of the endoplasmic reticulum. J Cell Sci. 2009;122(Pt 10):1626-1636.

27. Plate L, et al. Small molecule proteostasis regulators that reprogram the ER to reduce extracellular protein aggregation. Elife. 2016;5:e15550.

28. Craft CM, Whitmore DH, Wiechmann AF. Cone arrestin identified by targeting expression of a functional family. $J$ Biol Chem. 1994;269(6):4613-4619.

29. John SK, Smith JE, Aguirre GD, Milam AH. Loss of cone molecular markers in rhodopsin-mutant human retinas with retinitis pigmentosa. Mol Vis. 2000;6:204-215.

30. Sakuma H, Murakami A, Fujimaki T, Inana G. Isolation and characterization of the human X-arrestin gene. Gene. 1998;224(12):87-95.

31. Haze K, et al. Identification of the G13 (cAMP-response-element-binding protein-related protein) gene product related to activating transcription factor 6 as a transcriptional activator of the mammalian unfolded protein response. Biochem J. 2001;355(Pt 1):19-28.

32. Thuerauf DJ, Marcinko M, Belmont PJ, Glembotski CC. Effects of the isoform-specific characteristics of ATF6 alpha and ATF6 beta on endoplasmic reticulum stress response gene expression and cell viability. J Biol Chem. 2007;282(31):22865-22878

33. Wu J, et al. ATF6alpha optimizes long-term endoplasmic reticulum function to protect cells from chronic stress. Dev Cell. 2007;13(3):351-364.

34. Yamamoto $\mathrm{K}$, et al. Transcriptional induction of mammalian ER quality control proteins is mediated by single or combined action of ATF6alpha and XBP1. Dev Cell. 2007;13(3):365-376.

35. Yoshida H, et al. Endoplasmic reticulum stress-induced formation of transcription factor complex ERSF including NF-Y (CBF) and activating transcription factors 6alpha and 6beta that activates the mammalian unfolded protein response. Mol Cell Biol. 2001;21(4):1239-1248

36. Collins ML, et al. A branched DNA signal amplification assay for quantification of nucleic acid targets below 100 molecules/ ml. Nucleic Acids Res. 1997;25(15):2979-2984.

37. Player AN, Shen LP, Kenny D, Antao VP, Kolberg JA. Single-copy gene detection using branched DNA (bDNA) in situ hybridization. J Histochem Cytochem. 2001;49(5):603-612.

38. Stempel AJ, Morgans CW, Stout JT, Appukuttan B. Simultaneous visualization and cell-specific confirmation of RNA and protein in the mouse retina. Mol Vis. 2014;20:1366-1373.

39. Wang F, et al. RNAscope: a novel in situ RNA analysis platform for formalin-fixed, paraffin-embedded tissues. J Mol Diagn. 2012;14(1):22-29.

40. Miyagi H, et al. Transcriptional regulation of VEGFA by the endoplasmic reticulum stress transducer OASIS in ARPE-19 cells PLoS One. 2013;8(1):e55155.

41. Wu J, et al. Inhibition of GPR40 protects MIN6 $\beta$ cells from palmitate-induced ER stress and apoptosis. J Cell Biochem. 2012;113(4):1152-1158.

42. Yoshikawa T, Ogata N, Izuta H, Shimazawa M, Hara H, Takahashi K. Increased expression of tight junctions in ARPE-19 cells under endoplasmic reticulum stress. Curr Eye Res. 2011;36(12):1153-1163.

43. Gallagher CM, et al. Ceapins are a new class of unfolded protein response inhibitors, selectively targeting the ATF6 $\alpha$ branch. Elife. 2016;5:e11878.

44. Sidrauski C, et al. Pharmacological brake-release of mRNA translation enhances cognitive memory. Elife. 2013;2:e00498.

45. Das I, et al. Preventing proteostasis diseases by selective inhibition of a phosphatase regulatory subunit. Science. 2015;348(6231):239-242.

46. Mendez AS, et al. Endoplasmic reticulum stress-independent activation of unfolded protein response kinases by a small molecule ATP-mimic. Elife. 2015;4:e05434.

47. Shoulders MD, et al. Stress-independent activation of XBP1s and/or ATF6 reveals three functionally diverse ER proteostasis environments. Cell Rep. 2013;3(4):1279-1292.

48. Hood DC, et al. ISCEV standard for clinical multifocal electroretinography (mfERG) (2011 edition). Doc Ophthalmol. 2012;124(1):1-13.

49. Grandjean JMD, Plate L, Morimoto RI, Bollong MJ, Powers ET, Wiseman RL. Deconvoluting stress-responsive proteostasis signaling pathways for pharmacologic activation using targeted RNA sequencing. ACS Chem Biol. 2019;14(4):784-795 